\title{
Optimal claim behaviour for third-party liability insurances with perfect information
}

\author{
N.P. Dellaert, J.B.G. Frenk and E. Voshol \\ Erasmus University, Rotterdam, Netherlands
}

Received August 1990

Revised March 1991

\begin{abstract}
In this paper we analyse the optimal claim behaviour of a policy holder having a third-party liability insurance in which one is allowed to decide at the end of an insurance year which damages occurred during that year should be claimed. This analysis can only be carried out in detail in case the damages are negative exponentially distributed. Moreover, we present some computational results using an existing bonusmalus system and a horizon of 10 and 25 years and compare these results with similar computations for a corresponding third-party liability insurance in which the policy holder has to decide within a limited time period after the accident to claim or not to claim.
\end{abstract}

Keywords: Optimal critical claim size, Markov decision processes, Order statistics

\section{Introduction}

An important feature of premium rating systems for vehicle insurance is the no-claim or bonus-malus principle. This principle is meant to reward policy holders for not having claims during a year; that is, to grant a bonus to a careful driver. A bonus principle affects the policy holder's decision whether or not to file a claim in a particular instance. An example of such a bonus-malus scheme operative in the Netherlands is given in Table 1.

In the case of a third-party liability insurance the policy holder is generally allowed some period (at least 24 hours) to decide to claim or not to claim a particular damage. After this time period he has to take a decision. Since the bonus-malus system depends on the number of claims in each year, his claim behaviour reflects his uncertainty about what might happen in the remaining part of the present insurance year. This system and in particular the optimal claim behaviour of a policy holder having imperfect information about the future is discussed in detail by Dellaert et al. (1990). In the same paper an overview of the existing literature on this topic is also presented. However, recently some Dutch insurance companies allow insurants without any additional cost and under the same bonus-malus scheme to decide at the end of an insurance year which damages occurred during that year should be claimed. This means that the policy holder has perfect information about the number of accidents and the corresponding damages during an insurance year at the moment he decides which damages to claim.

The purpose of this paper is to analyse the optimal claim behaviour of a policy holder having complete information at his decision moments and to compare his corresponding costs with the costs for an optimally claiming policy holder operating under an imperfect information system as discussed in Dellaert et al. (1990). In Section 2 a mathematical description of a third-party liability insurance with perfect information is given. Due

Table 1

Percentages of the basic premium by bonus-malus class.

\begin{tabular}{llrlll}
\hline $\begin{array}{l}\text { Bonus- } \\
\text { malus } \\
\text { class }\end{array}$ & $\begin{array}{l}\text { Premium as } \\
\text { percentage } \\
\text { of the basic } \\
\text { premium }\end{array}$ & \multicolumn{4}{l}{$\begin{array}{l}\text { New bonus-malus } \\
\text { class after } \ldots \text { claims }\end{array}$} \\
\cline { 3 - 6 } & 0 & 1 & 2 & $\geq 3$ \\
\hline 14 & 30.0 & 14 & 9 & 5 & 1 \\
13 & 32.5 & 14 & 8 & 4 & 1 \\
12 & 35.0 & 13 & 8 & 4 & 1 \\
11 & 37.5 & 12 & 7 & 3 & 1 \\
10 & 40.0 & 11 & 7 & 2 & 1 \\
9 & 45.0 & 10 & 6 & 1 & 1 \\
8 & 50.0 & 9 & 5 & 1 & 1 \\
7 & 55.0 & 8 & 4 & 1 & 1 \\
6 & 60.0 & 7 & 3 & 1 & 1 \\
5 & 70.0 & 6 & 2 & 1 & 1 \\
4 & 80.0 & 5 & 1 & 1 & 1 \\
3 & 90.0 & 4 & 1 & 1 & 1 \\
2 & 100.0 & 3 & 1 & 1 & 1 \\
1 & 120.0 & 2 & 1 & 1 & 1 \\
\hline
\end{tabular}


to the analytical intractability of the distribution of sums of order statistics we focus our attention in Section 3 on the case where the damages are independent and negative exponentially distributed. Finally in Section 4 we present an example of a third-party liability insurance with and without perfect information and compare both systems.

\section{The model}

In order to model the problem discussed in the previous section as a discrete time Markov decision process we have to define:

- the set $\mathscr{T}$ of decision moments,

- the decision set $\mathscr{D}$,

- the state space $\mathscr{S}$.

Clearly, if we assume that the insurance will continue for $T$ years $(T<\infty)$ the policy holder has to take decisions at the end of each year and hence $\mathscr{T}:=\{t \mid t=1,2, \ldots, T\}$.

Moreover, by Table 1 the policy holder has the option at the end of each insurance year in which $k$ accidents occurred to claim $i$ damages with $0 \leq i \leq \min (2, k)$ or if $k>2$ to claim all the damages. Therefore $\mathscr{D}=\{0,1,2,3\}$ with $i:=$ claim $i$ damages, $0 \leq i \leq 2$ and $3:=$ claim all damages. Of course, since the insurance premium to be paid for the next year only depends on the number of filed claims at the end of the present year the policy holder claiming $i$ damages claims the $i$ biggest damages. We now introduce the state space. Before doing this we define for $1 \leq t \leq T$ the following random variables:

$\underline{N}_{t} \quad:=$ number of accidents in year $t$,

$\underline{X}_{j t}:=$ amount of damage of $j$ th accident in insurance year $t, 0 \leq j \leq \underline{N}_{t}$,

$\underline{X}_{j: N_{t}}:=$ the $j$ th smallest amount of damage occurring in insurance year $t$, i.e. the $j$ th order statistic of the sample $\underline{X}_{t}, 0 \leq j \leq \underline{N}_{t}$.

Clearly $\underline{X}_{0 t}=0$ and

$0=\underline{X}_{0: \underline{N}_{t}} \leq \underline{X}_{1: \underline{N}_{t}} \leq \underline{X}_{2: \underline{N}_{t}} \leq \ldots \leq \underline{X}_{\underline{N}_{t}: \underline{N}_{t}}$.

Assume now that the random variables $\underline{N}_{t}$, $1 \leq t \leq T$, are independent and identically distributed with $P\left\{\underline{N}_{t}=k\right\}=p_{k}, k \geq 0$, and that the same holds for the sequence $\underline{X}_{j t}, 1 \leq j \leq \underline{N}_{t}, 1 \leq t$ $\leq T$, with distribution $G$ satisfying $G(0)=0$. Furthermore, let the sequences $\left\{\underline{N}_{t}: 1 \leq t \leq T\right\}$ and $\left\{\underline{X}_{j t}: 0 \leq j \leq \underline{N}_{t}, 1 \leq t \leq T\right\}$ be independent of each other. In the remainder we will omit the subscript $t$ and denote the realisations of $\underline{N}$ and $\underline{X}_{j: \underline{N}}$ by $k$, resp. $x_{j}$. To complete our description define:

$j \quad:=$ the policy holder's bonus-malus class, $1 \leq j \leq J$,

$\pi(j):=$ premium to be paid at the beginning of an insurance year if the policy holder enters bonus-malus class $j$,

$b_{i}(j):=$ bonus-malus class the policy holder enters next year if he is at the moment in bonus-malus class $j$ and decides to take action $i \in \mathscr{D}$ at the end of the present year.

By the above definitions an elements $s$ of the state space $S$ is given by

$s=\left(x_{0}, x_{1}, \ldots, x_{k}, j\right), k \geq 0,1 \leq j \leq J$,

where $x_{0}=0$ (in the remainder we will omit $x_{0}$ in the state description).

Define now for every $1 \leq t \leq T$;

$V_{t}\left(x_{1}, \ldots, x_{k}, j\right):=$ minimal expected discounted cost for the policy holder from the end of year $t$ until the end of the insurance period, if this policy holder observed state $\left(x_{1}, \ldots, x_{k}, j\right)$ at the end of insurance year $t$ just before he has to decide which claims to file,

and

$V_{0}(j):=$ minimal expected discounted cost for the policy holder of the total insurance period it this policy holder enters bonus-malus class $j$ at the beginning of his insurance.

\section{Clearly}

$V_{T}\left(x_{1}, \ldots, x_{k}, j\right)=0$

and

$V_{0}(j)=\pi(j)+\beta \mathbb{E} V_{1}\left(\underline{X}_{1: \underline{N}}, \ldots, \underline{X}_{\underline{N}: \underline{N}}, j\right)$

with $\mathbb{E}$ denoting the mathematical expectation symbol. 
If we define $a_{i}(t), 0 \leq i \leq 3$, by

$$
\begin{aligned}
a_{i}(t):= & \pi\left(b_{i}(j)\right) \\
& +\beta \mathbb{E} V_{t+1}\left(\underline{X}_{1: \underline{N}}, \ldots, \underline{X}_{\underline{N}: \underline{N}}, b_{i}(j)\right)
\end{aligned}
$$

we obtain for the years $1 \leq t \leq T-1$ the following set of backward equations:

$$
\begin{aligned}
& V_{t}\left(x_{0}, \ldots, x_{k}, j\right) \\
& \quad=\min \left\{\sum_{n=0}^{l_{i, k}(t)} x_{n}+a_{i}(t): 0 \leq i \leq \min (k, 3)\right\}
\end{aligned}
$$

with

$l_{i, k}(t):=\left\{\begin{array}{lll}k-i & \text { if } & i<\min (k, 3) \\ 0 & \text { if } & i=\min (k, 3)\end{array}\right.$.

From these backward equations we see that an optimal decision is easy to determine at the end of insurance year $t$ if the values $\mathbb{E} V_{t+1}\left(\underline{X}_{1: \underline{N}}, \ldots\right.$, $\left.\underline{X}_{N: \underline{N}}, j\right)$ are known for every $j$. Hence to solve the model we have to compute these expectations. Therefore we shall take a closer look at the structure of the backward equations and derive some general properties of $\mathbb{E} V_{l}\left(\underline{X}_{1: \underline{N}}, \ldots, \underline{X}_{\underline{N}: \underline{N}}, j\right), 1 \leq$ $t \leq T-1$.

First of all we observe that for every fixed $t$,

$a_{0}(t) \leq a_{1}(t) \leq a_{2}(t) \leq a_{3}(t)$.

These inequalities hold since the premium to be paid for entering bonus-malus class $b_{i}(j)$ is nondecreasing in $i$, while at the same time, being in bonus-malus class $b_{i}(j)$ in year $t$, the minimal expected discounted cost from year $t+1$ until the cnd of the insurance period is also non-decreasing in $i$. (Hint: use induction with respect to $t$.)

Moreover, it also follows for every $t \geq 1$ that

$$
\begin{aligned}
\mathbb{E} V_{l} & \left(\underline{X}_{1: \underline{N}}, \ldots, \underline{X}_{\underline{N}: \underline{N}}, j\right) \\
= & \sum_{k=0}^{\infty} \mathbb{E}\left(V_{t}\left(\underline{X}_{1: \underline{N}}, \ldots, \underline{X}_{\underline{N}: \underline{N}}, j\right) \mid \underline{N}=k\right) \\
& \times P\{\underline{N}=k\} \\
= & \sum_{k=0}^{\infty} \mathbb{E} V_{t}\left(\underline{X}_{1: k}, \ldots, \underline{X}_{k: k}, j\right) p_{k} .
\end{aligned}
$$

Hence by (2.1) and (2.2), in order to compute
$\mathbb{E} V_{l}\left(\underline{X}_{1: \underline{N}}, \ldots, \underline{X}_{\underline{N}: \underline{N}}, j\right)$, we have to calculate

$$
\begin{aligned}
& \mathbb{E} V_{t}\left(\underline{X}_{1: k}, \ldots, \underline{X}_{k: k}, j\right) \\
& \quad=\mathbb{E}\left(\min _{0 \leq i \leq k}\left(a_{i}(t)+\sum_{n=0}^{k-i} \underline{X}_{n: k}\right)\right)
\end{aligned}
$$

if $0 \leq k \leq 2$ and

$$
\begin{aligned}
& \mathbb{E} V_{t}\left(\underline{X}_{1: k}, \ldots, \underline{X}_{k: k}, j\right) \\
& \quad=\mathbb{E}\left(\min \left(\min _{0 \leq i \leq 2}\left(a_{i}(t)+\sum_{n=0}^{k-i} \underline{X}_{n: k}\right), a_{3}\right)\right)
\end{aligned}
$$

if $k \geq 3$.

For the above expectations one can derive the following inequalities.

Lemma 2.1. For every bonus-malus class $j, k \geq 3$ and $1 \leq t \leq T$ we have

$$
\begin{aligned}
& \mathbb{E} V_{t}\left(\underline{X}_{1: k}, \ldots, \underline{X}_{k: k}, j\right) \\
& \quad \leq \mathbb{E} V_{t}\left(\underline{X}_{1: k+1}, \ldots, \underline{X}_{k+1: k+1}, j\right) .
\end{aligned}
$$

Proof. Let $\underline{X}_{1}, \ldots, \underline{X}_{k}$ be a random sample from a distribution $G$ and denote by $\underline{X}_{1: k} \leq \ldots \leq \underline{X}_{2: k}$ $\leq \ldots \leq \underline{X}_{k: k}$ the corresponding sequence of order statistics. If $\underline{X}_{k+1}$ is an additional drawing from the same probability distribution and $\underline{X}_{1: k+1} \leq$ $\underline{X}_{2: k+1} \leq \cdots \leq \underline{X}_{k+1: k+1}$ is the newly formed sequence of order statistics one can easily verify that

$\sum_{n=1}^{p+1} \underline{X}_{n: k+1} \geq \sum_{n=1}^{p} \underline{X}_{n: k}$

for every $1 \leq p \leq k$. Hence the desired result follows by $(2.5)$.

By Lemma 2.1 it is now possible to derive the following lower and upper bounds.

Lemma 2.2. For every bonus-malus class $1 \leq j \leq J$ we have

$$
\begin{aligned}
& \mathbb{E} V_{t}\left(\underline{X}_{1: 3}, \ldots, \underline{X}_{3: 3}, j\right) P\{\underline{N} \geq 4\} \\
& \quad \leq \sum_{k=4}^{\infty} \mathbb{E} V_{t}\left(\underline{X}_{1: k}, \ldots, \underline{X}_{k: k}, j\right) p_{k} \\
& \quad \leq a_{3} P\{\underline{N} \geq 4\} .
\end{aligned}
$$

Proof. By (2.5) clearly

$\mathbb{E} V_{t}\left(\underline{X}_{1: k}, \ldots, \underline{X}_{k: k}, j\right) \leq a_{3}$ 
for every $k \geq 3$ and hence

$$
\sum_{k=4}^{\infty} \mathbb{E} V_{t}\left(\underline{X}_{1: k}, \ldots, \underline{X}_{k: k}, j\right) p_{k} \leq a_{3} P\{\underline{N} \geqslant 4\}
$$

Moreover, by Lemma 2.1 the other inequality follows.

It is clear by the above lemma and (2.3) that a lower and upper bound on $\mathbb{E} V_{t}\left(\underline{X}_{1: \underline{N}}, \ldots, \underline{X}_{\underline{N}: \underline{N}}\right.$, $j$ ) can be derived. Since this derivation is obvious we will omit it.

In the next section we will discuss an example of a distribution $G$ for which it is possible to derive an analytical expression for the upper and lower bound.

\section{Negative exponential distribution amount of damage}

In this section we assume that $\underline{X}_{1}, \ldots, \underline{X}_{k}$ is a random sample from a negative exponential distribution with parameter $\mu>0$. Then it is possible to calculate $\mathbb{E} V_{t}\left(\underline{X}_{1: k}, \ldots, \underline{X}_{k K k}, j\right)$ for every $k \leq 3$ and so by the remark after Lemma 2.2 we obtain an approximation of $\mathbb{E} V_{t}\left(\underline{X}_{1: \underline{N}}, \ldots, \underline{X}_{\underline{N}: \underline{N}}, j\right)$ with computable lower and upper bounds. The computation is carried out using the computer system Maple, which is a system of symbolic mathematical computation (cf. [3]). However, in order to apply Maple we need to rewrite the expectations in a proper form making use of the properties of the exponential distribution. This procedure of rewriting the expectations and the corresponding mathematical manipulations are carried out in detail in the Appendix of Dellaert et al. (1990) and so we only mention the results.

Define now

$$
\begin{aligned}
& F_{1}(a, b, \mu):=\mathbb{E}\left(\min \left(a+\underline{X}_{1: 1}, b\right)\right) \\
& F_{2}(a, b, c, \mu) \\
& \quad:=\mathbb{E}\left(\min \left(a+\underline{X}_{1: 2}+\underline{X}_{2: 2}, b+\underline{X}_{1: 2}, c\right)\right)
\end{aligned}
$$

and

$$
\begin{aligned}
F_{k}(a, b, c, d, \mu) & \\
:=\mathbb{E}\left(\operatorname { m i n } \left(a+\sum_{n=1}^{k} \underline{X}_{n: k},\right.\right. & b+\sum_{n=1}^{k-1} \underline{X}_{n: k}, c \\
& \left.\left.+\sum_{n=1}^{k-2} \underline{X}_{n: k}, d\right)\right)
\end{aligned}
$$

for every $k \geq 3$.

Observe, if one assigns to the variables $a, b, c$ and $d$ the values $a_{i}(t), 0 \leq i \leq 3$, then the function $F_{k}\left(a_{0}, a_{1}, a_{2}, a_{3}, \mu\right)$ equals the expected minimal cost from year $t$ until the end of the insurance period if the policy holder is in bonusmalus class $j$ at the beginning of the forthcoming insurance year and the number of accidents in that new insurance year is equal to $k$.

Since the random variables $\underline{X}_{i}, i \geq 1$, are independent and negative exponentially distributed with parameter $\mu>0$ it is not difficult to verify that

$$
\begin{aligned}
& F_{1}(a, b, \mu)=\frac{1}{\mu} F_{1}(\mu a, \mu b, 1), \\
& F_{2}(a, b, c, \mu)=\frac{1}{\mu} F_{2}(\mu a, \mu b, \mu c, 1)
\end{aligned}
$$

and

$F_{k}(a, b, c, d, \mu)=\frac{1}{\mu} F_{k}(\mu a, \mu b, \mu c, \mu d, 1)$

for every $k \geq 3$.

Hence we can assume without loss of generality that $\mu=1$ and so it is sufficient to calculate the functions $F_{k}(a, b, c, d, 1)$. Starting with $k=1$ it is easy to verify that

$$
F_{1}(a, b, 1)= \begin{cases}a+1-\exp (a-b) & \text { if } 0 \leq a<b \\ b & \text { if } a \geq b\end{cases}
$$

Table 2

Results of the computation of $F_{2}(a, b, c, 1)$.

\begin{tabular}{lc}
\hline & $F_{2}(a, b, c, 1)$ \\
\hline$c \leq \min (a, b)$ & $c$ \\
$b \leq \min (a, c)$ & $0.5+b-0.5 \exp (2 b-2 c)$ \\
$a \leq c \leq b$ & $2+a+(-2+a-c) \exp (a-c)$ \\
$a \leq b \leq c$ & $2+a-2 \exp (a-b)+0.5 \exp (2 a-2 b)$ \\
$\quad \& b-a \leq c-b$ & $\quad-0.5 \exp (2 b-2 c)$ \\
$a \leq b \leq c$ & $2+a-2 \exp (a-b)$ \\
$\& c-b \leq b-a$ & $+(a-2 b+c) \exp (a-c)$ \\
\hline
\end{tabular}


Moreover, for $k=2$ the results are shown in Table 2.

Finally, for $k=3$ the long and complicated formulas are shown in Dellaert, Frenk and Voshol (1990) and are therefore omitted.

In Section 4 the model and the formulas derived in this section will be applied to an existing third-party liability insurance. It will be shown that the difference between the lower and upper bound on $\mathbb{E} V_{t}\left(\underline{X}_{1: N}, \ldots, \underline{X}_{N: N}, j\right)$ as discussed after Lemma 2.2 is negligible over a period of 10 or 25 years and therefore either the computable lower or upper bound can be used to determine the minimal expected cost of the policy holder and his corresponding optimal decisions.

\section{Computational results}

The model presented in Section 3 will be applied to the bonus-malus system given in Table 1 . As in Dellaert et al. (1990) we divide an insurance year in $N$ equal periods and assume that the probability $p_{n}$ to have an accident during period $n$ is equal to $\lambda / N$. To compare our results with the results found in Dellaert et al. (1990) we take the probability $p_{k}$ of having $k$ accidents during an insurance year equal to

$$
\left(\begin{array}{c}
N \\
k
\end{array}\right)\left(\frac{\lambda}{N}\right)^{k}\left(1-\frac{\lambda}{N}\right)^{N-k} \text {. }
$$

Usually the number of accidents during an insurance year is assumed to be Poisson-distributed, but the above choice is only little different when $\lambda$ is fixed and $N$ is big [cf. Hogg and Craig (1978)]. We assume the amount of damage $\underline{X}_{i}$ to be negative exponentially distributed with parameter $\mu$. For reasons of simplicity the parameters $\lambda$ and $\mu$ are constant throughout the entire insurance period.

For a third-party liability insurance a value of $\lambda=0.1$ accidents per year is reasonable. We take the value of $\mu=1800^{-1}$, that is, the expected amount of damage equals Dfl.1800. The basic premium is equated to Dfl.1000, and the annual interest rate to 5 percent. Therefore the annual discount rate equals $\beta=0.95238$.

The lower and upper bounds on $\mathbb{E} V_{l}\left(\underline{X}_{1}: \underline{N}, \ldots\right.$, $\left.\underline{X}_{\underline{N}: \underline{N}}, j\right)$ discussed after Lemma 2.2 are calculated for the above values for a period of 10 and 25 years. It is found that in both cases the difference between these bounds is even smaller than Dfl.0.05. Since this difference is negligible, it is legitimate to use either the computable lower or upper bound for the expectation given after Lemma 2.2. The upper bounds for a period of 10 years are given in Table 3.

Since at decision moments the number $k$ of accidents and the corresponding damages are known the policy holder can deduce his optimal claim behaviour from Table 3 and the expression (2.2).

Table 3

Upper bound on $\mathbf{E} V_{t}\left(\underline{X}_{1: \underline{N}}, \ldots, \underline{X}_{\underline{N}}: \underline{N}, j\right)$ for a horizon of 10 years, i.e. $T=10$ and $0 \leq t \leq 9$.

\begin{tabular}{llllllllllr}
\hline $\begin{array}{l}\text { Bonus- } \\
\text { malus } \\
\text { class } j\end{array}$ & $t=0$ & $t=1$ & $t=2$ & $t=3$ & $t=4$ & $t=5$ & $t=6$ & $t=7$ & $t=8$ & $t=9$ \\
\hline 14 & 2682 & 2501 & 2267 & 2022 & 1765 & 1497 & 1216 & 925 & 624 & 315 \\
13 & 2721 & 2516 & 2282 & 2036 & 1779 & 1510 & 1228 & 935 & 632 & 319 \\
12 & 2781 & 2553 & 2318 & 2072 & 1814 & 1543 & 1260 & 966 & 659 & 342 \\
11 & 2876 & 2626 & 2390 & 2143 & 1882 & 1609 & 1323 & 1024 & 710 & 369 \\
10 & 2984 & 2714 & 2477 & 2228 & 1965 & 1689 & 1399 & 1093 & 758 & 393 \\
9 & 3148 & 2833 & 2594 & 2342 & 2075 & 1794 & 1497 & 1169 & 809 & 420 \\
8 & 3364 & 3007 & 2764 & 2508 & 2236 & 1948 & 1628 & 1275 & 891 & 474 \\
7 & 3627 & 3230 & 2984 & 2721 & 2442 & 2131 & 1787 & 1411 & 999 & 528 \\
6 & 3932 & 3499 & 3246 & 2977 & 2676 & 2341 & 1974 & 1571 & 1106 & 582 \\
5 & 4326 & 3807 & 3547 & 3257 & 2933 & 2576 & 2184 & 1729 & 1213 & 625 \\
4 & 4801 & 4201 & 3921 & 3609 & 3264 & 2884 & 2441 & 1938 & 1371 & 742 \\
3 & 5320 & 4641 & 4341 & 4008 & 3640 & 3214 & 2726 & 2175 & 1560 & 834 \\
2 & 5875 & 5119 & 4799 & 4445 & 4034 & 3564 & 3033 & 2437 & 1735 & 926 \\
1 & 6556 & 5624 & 5284 & 4890 & 4440 & 3931 & 3358 & 2684 & 1908 & 1018 \\
\end{tabular}


In Table 4 the minimal expected cost for the policy holder with perfect information is listed for eight different cases. These costs are calculated for different time horizons ( 10 and 25 years), different values of $\lambda(\lambda=0.1$ and $\lambda=0.2)$, and different values of $\beta[\beta=0.95238$ (discounted), and $\beta=1.0$ (undiscounted)]. Similar cost calculations for the model with imperfect information [Dellaert et al. (1990)] are performed and the corresponding differences in cost compared with the model having perfect information are listed in Table 5. Clearly the minimal expected cost for a policy holder with perfect information is always smaller than in the case of imperfect information. This confirms our intuition that the additional (free) information enables the policy holder to take better decisions and therefore lowers his expected cost. Only, as shown in Table 5, the benefit from this information is very small.

Up to this point the lower and upper bounds on $\mathbb{E} V_{t}\left(\underline{X}_{1: \underline{N}}, \ldots, \underline{X}_{N: N}, j\right)$ are compared only for relevant values of $\lambda$. In the non-discounted model, where $\beta=1.0$, we have computed the lower and upper bounds also for values of $\lambda$ up to 1.0. The difference between these bounds grows rapidly from $0.001 \%$ of the total cost when $\lambda=0.1$ to approximately $0.30 \%$ when $\lambda=1.0$. The actual difference is smaller than Dfl.72 over a period of 25 years and smaller than Dfl. 32 over a period of 10 years. So the computable lower and upper bound are relatively close for extreme values of $\lambda$.
Table 5

Extra cost due to imperfect information.

\begin{tabular}{rllllllll}
\hline$T$ & 10 & 25 & 10 & 25 & 10 & 25 & 10 & 25 \\
$\lambda$ & 0.1 & 0.1 & 0.2 & $\mathbf{0 s . 2}$ & $\mathbf{0 . 1}$ & $\mathbf{0 . 1}$ & 0.2 & 0.2 \\
$\mathbf{d} / \mathbf{u d}$ & $\mathbf{d}$ & $\mathbf{d}$ & $\mathbf{d}$ & $\mathbf{d}$ & $\mathbf{u d}$ & $\mathbf{u d}$ & $\mathbf{u d}$ & $\mathbf{u d}$ \\
\hline BM class 14 & 12 & 25 & 18 & 46 & $\mathbf{1}$ & 7 & 6 & 27 \\
13 & 12 & 26 & 19 & 47 & 2 & 8 & 7 & 27 \\
12 & 12 & 26 & 19 & 47 & 1 & 7 & 6 & 26 \\
11 & 12 & 27 & 19 & 48 & 2 & 7 & 6 & 27 \\
10 & 14 & 27 & 21 & 49 & 2 & 7 & 7 & 27 \\
9 & 14 & 28 & 22 & 51 & 2 & 8 & 7 & 27 \\
8 & 15 & 30 & 23 & 52 & 2 & 7 & 6 & 26 \\
7 & 16 & 31 & 24 & 54 & 2 & 7 & 5 & 25 \\
6 & 18 & 31 & 27 & 58 & 1 & 7 & 6 & 25 \\
5 & 19 & 35 & 30 & 61 & 1 & 6 & 8 & 26 \\
4 & 23 & 38 & 36 & 69 & 3 & 8 & 11 & 30 \\
3 & 25 & 42 & 39 & 74 & 4 & 9 & 15 & 34 \\
2 & 27 & 44 & 41 & 77 & 4 & 10 & 16 & 37 \\
1 & 28 & 46 & 40 & 79 & 4 & 10 & 15 & 37 \\
& & & & & & & &
\end{tabular}

Hence, even for these extreme values the computable lower or upper bound can be used for the minimal expected cost. In the remainder we will use the upper bound.

Next the minimal expected cost for the policy holder with perfect information is computed for values of $\lambda=0.1$ up to $\lambda=1.0$ for a time horizon of 25 years in the non-discounted model $(\beta=1.0)$. These values are compared with the results from the model with imperfect information. The minimal expected cost for a policy holder with perfect information is always smaller than in the

Table 4

Minimal expected cost for the total insurance period with perfect information, in Dfl.

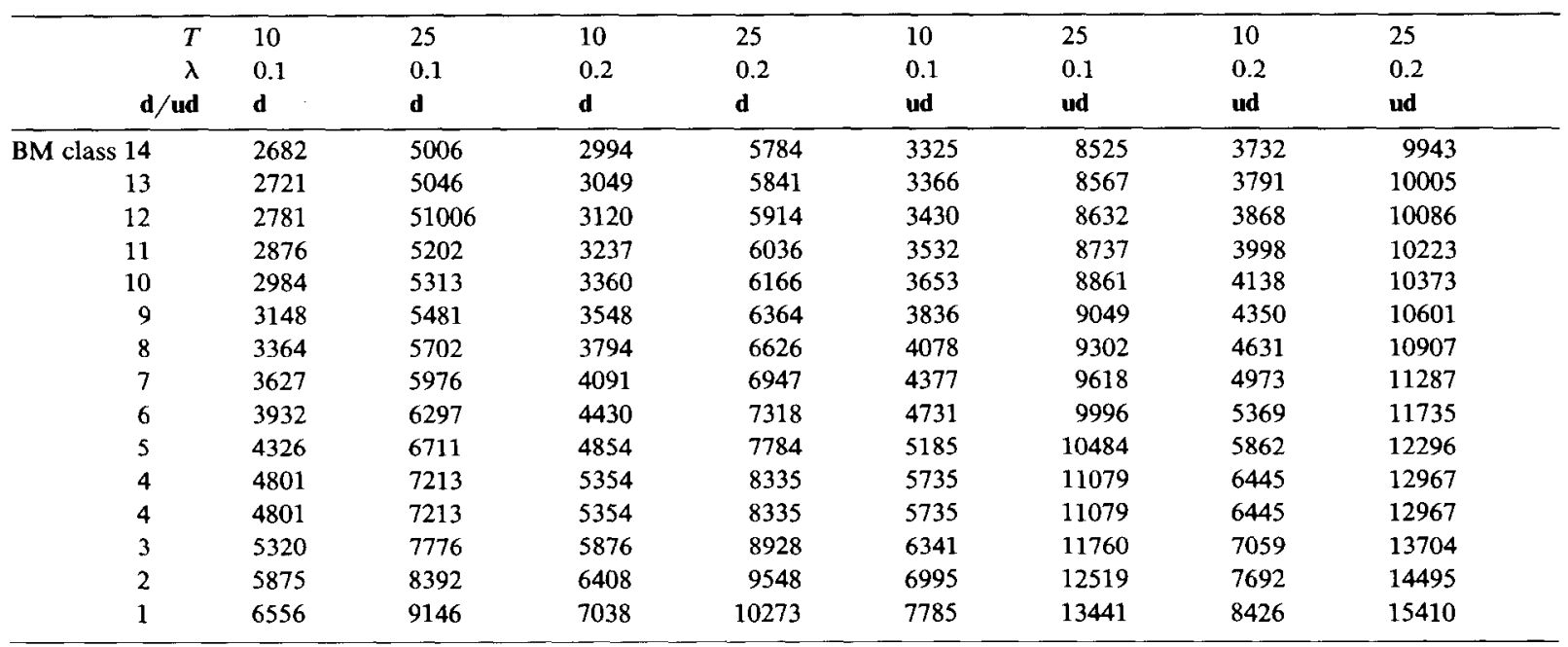


case of imperfect information. It is found that the difference in cost between both models increases as $\lambda$ increases, but remains smaller than Dfl.300 (which is less than $1 \%$ of the minimal expected cost). So the additional (free) information enables the policy holder to take better decisions and therefore lowers his expected cost, but still the benefit is very small.

\section{Acknowledgement}

The authors like to thank the anonymous referee and R. Kaas for their constructive comments and criticism.

\section{References}

Dellaert, N.P., J.B.G. Frenk and E. Voshol (1990). Optimal claim behaviour for third-party liability insurances with perfect information. Report 9071/A. Econometric Institute, Erasmus University, Rotterdam.

Dellaert, N.P., J.B.G. Frenk, A. Kouwenhoven and B.S. van der Laan (1990). Optimal claim behaviour for third-party liability insurances or to claim or not to claim: That is the question. Insurance: Mathematics and Economics 9, 59-76.

Hogg, R.V. and A.T. Craig (1978). Introduction to Mathematical Statistics, Fourth edition. Macmillan Publishing Co. Inc., New York.

Maple Reference Manual, 5th edition (1988). Symbolic Computation Group Dep. of Computer Science, Univ. of Waterloo, Waterloo, Ontario, Canada N2L 3G1. 